\title{
GRANULOMA PIÓGENO EN TAPONES LAGRIMALES SMART PLUG
}

\section{PYOGENIC GRANULOMA FOLLOWING SMART PLUG INSERTION}

PASTOR-PASCUAL F ${ }^{1}$, AVIÑÓ-MARTÍNEZ J² ${ }^{2}$ ESPAÑA-GREGORI E ${ }^{3}$, ALCOCER-YUSTE P ${ }^{1}$

\section{RESUMEN}

Caso clínico: Mujer de 65 años, diagnosticada de ojo seco, acude por aparición de tumoración en canto interno con sensación de cuerpo extraño. Tres años antes se le habían implantado tapones lagrimales SmartPlug en ambos puntos lagrimales inferiores. Se apreció un granuloma en el punto lagrimal inferior izquierdo que se extirpó. Dos semanas después presentó una recidiva lo que obligó a extraer el implante quedándose desde entonces asintomática. Discusión: Estamos ante un granuloma piógeno en un implante punctal SMART PLUG, complicación descrita clásicamente con los implantes de silicona, debido posiblemente a la irritación crónica de los detritus acumulados. Esta complicación obliga a extraer los tapones.

Palabras clave: Ojo seco, tapones lagrimales, Smart Plug, granuloma piógeno, parche punctal.

\begin{abstract}
Case report: We report the case of a 65 -year-old woman with dry eye syndrome who was referred because of a red mass in the internal left canthus. Three years previously two Smart Plugs had been introduced into both lacrimal punctums of that eye. We diagnosed a pyogenic granuloma and removed it. Two weeks later a new granuloma developed so both the granuloma and the punctal plug were removed. The patient became asymptomatic following this latter procedure.

Discussion: A pyogenic granuloma in a Smart Plug punctum is described. This rare complication is generally associated with the use of silicone punctal plugs, being possibly caused by the chronic irritation of the accumulated detritus and necessitating removal of the plug (Arch Soc Esp Oftalmol 2007; 82: 653-656).
\end{abstract}

Key words: Dry eye, punctal plugs, Smart Plug, pyogenic granuloma, punctal occlusion.

\footnotetext{
Recibido: 8/8/06. Aceptado: 25/9/07.

Servicio de Oftalmología. Hospital Universitario La Fe. Valencia. España.

1 Licenciado en Medicina.

2 Doctor en Medicina.

3 Doctor en Medicina. Departamento de Óptica. Universidad de Valencia.

Correspondencia:

Francisco Pastor Pascual

Fundación Oftalmológica del Mediterráneo

Bifurcación Pío Baroja-General Avilés, s/n

46015 Valencia

España

E-mail: oftpaco@yahoo.es
} 


\section{INTRODUCCIÓN}

El ojo seco es una patología de etiología variada y compleja. Los síntomas de sequedad, enrojecimiento, sensación de tierra o de cuerpo extraño son derivados del desequilibrio en la cantidad o calidad de las lágrimas.

Se han propuesto distintos tratamientos para dicha patología. El más utilizado es el uso de lágrimas artificiales. En casos de clínica refractaria a las lágrimas artificiales, una de las posibilidades es la oclusión del punto lagrimal, con lo que aumenta el tiempo de permanencia de la lagrima (1). El punto puede ser taponado irreversiblemente con cauterio, tratamiento láser o un injerto de conjuntiva (2) o bien de forma reversible con un dispositivo llamado tapón lagrimal.

El uso de los tapones lagrimales se ha demostrado como una opción efectiva en el tratamiento del ojo seco. Existen distintos tipo de tapones como los tapones de silicona y el SMART-PLUG (Mednnium Inc, 15350 Barranca Parkway, Irvenie CA, USA). Los tapones de silicona se utilizan mayoritariamente desde hace años. Se han descrito como complicaciones de estos tapones la extrusión espontánea del tapón o la formación de un granuloma piógeno $(3,4)$, complicaciones ambas que obligan a la extracción quirúrgica del mismo.

Los tapones SMART-PLUG son unos implantes con un diseño que simplifica la inserción (no precisa la dilatación del punto lagrimal) y elimina la posibilidad de la irritación ocular debida al roce de la punta del tapón. Se trata de un material acrílico que altera sus propiedades físicas, pasando de ser sólido rígido a un gel suave en respuesta a un aumento de temperatura. Pasa de tener un diámetro de 0,4 a $1 \mathrm{~mm}$. Así su introducción se ve facilitada y al transformarse, el gel se adapta a la forma del canalículo expandiéndose. El material acrílico hidrofóbico termosensible es totalmente biocompatible por lo que no causa toxicidad.

\section{CASO CLÍNICO}

Mujer de 65 años de edad que acude a Consultas Externas de Oftalmología por sensación de cuerpo extraño y tumoración rojo violácea en el canto interno izquierdo. A la exploración se observa la presencia de un granuloma que asoma por el punto lagrimal inferior.
Se trataba de una paciente que estaba siendo controlada desde hacía 4 años en Consultas Externas de Oftalmología por presentar un síndrome de ojo seco que se objetivó en las distintas exploraciones realizadas en las sucesivas visitas presentando un test de Schirmer menor de 5 mm., un BUT de entre 1 y 2 segundos y la práctica inexistencia de menisco lagrimal.

Había recibido diversos tratamientos con distintos tipos de lubricantes sin notar mejoría significativa. Ante la persistencia de sus molestias crónicas se le implantaron unos tapones lagrimales SMARTPLUG en canalículo inferior de ambos ojos, y se aconsejó continuar con el tratamiento lubricante (carmelosa 0,5\%), con lo que experimentó una leve mejoría que remitió a los 6 meses.

Ante este empeoramiento de su molestias se le pautó Pilocarpina oral $5 \mathrm{mg}$ cada 8 horas (Salagen ${ }^{\circledR}$ ) advirtiendo un alivio temporal. Posteriormente, una año después del implante se le suspendió la pilocarpina por la ineficacia del mismo y se le implantó un nuevo SMART-PLUG en el canalículo superior del ojo izquierdo sin apreciar mejora. Además recibió tratamiento con colirio de suero autólogo primero y de ciclosporina tópica posteriormente sin beneficio clínico por lo que se retiraron dichos colirios recibiendo únicamente tratamiento con lágrimas artificiales.

A los tres años del primer implante la paciente acudió al servicio de Urgencias por presentar un granuloma en el canalículo inferior del ojo izquierdo que se extirpó en ese momento. Dos semanas más tarde acude a Consultas externas por presentar una recidiva del granuloma (fig. 1).

Se procedió a la extracción del implante punctal (fig. 2) mediante canaliculotomía transcutánea en quirófano, realizando además desbridamiento y limpieza del canalículo. No se implantó ninguna sonda o dispositivo canalicular. Tras la cirugía desapareció el cuadro infeccioso, no apreciándose hasta la fecha recidiva del granuloma

\section{DISCUSIÓN}

Por lo tanto es un caso de un granuloma piógeno en un implante punctal SMART-PLUG. Los granulomas piógenos son una complicación descrita en los implantes lagrimales, normalmente en los implantes de silicona. Hasta la fecha hay escasos casos descritos en la literatura de la aparición de dicha complicación en los tapones lagrimales 


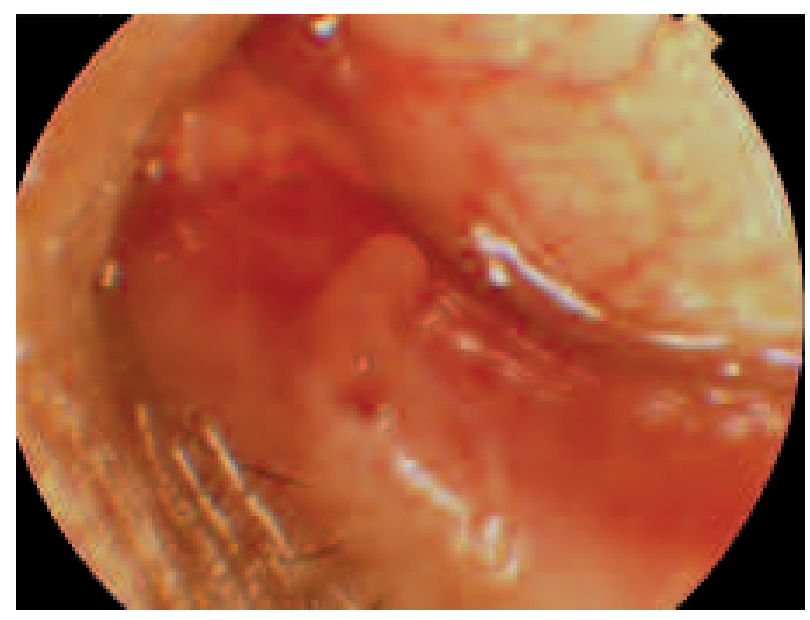

Fig. 1: Granuloma piógeno.

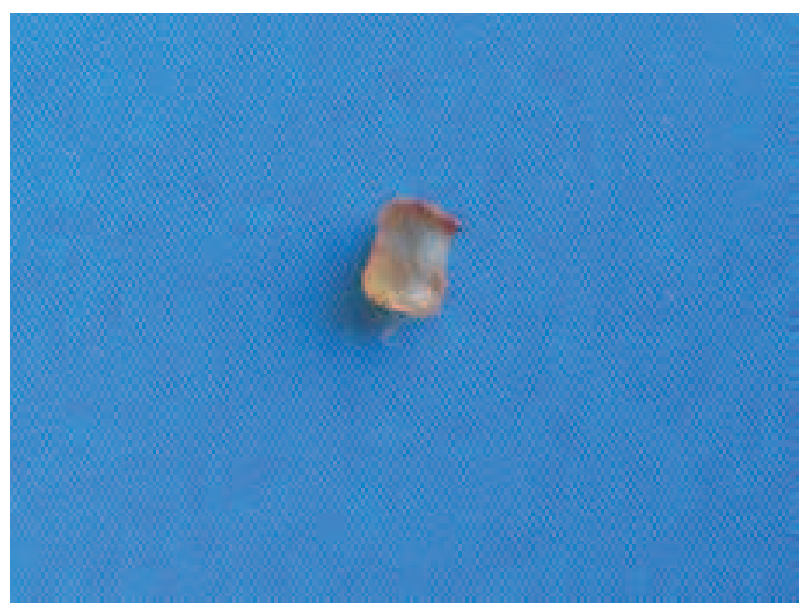

Fig. 2: Aspecto del tapón lagrimal tras su extracción.
SMART PLUG (5), debido a que se trata de un material acrílico biocompatible.

El hecho de que haya aparecido en el ojo izquierdo podría ser debido a que al ocluir ambos puntos lagrimales ocasionaría un cúmulo de detritus que podría producir una irritación crónica que favorecería la formación del granuloma con el transcurso del tiempo.

A pesar de la aparente inocuidad del procedimiento de la implantación de estos tapones, debemos conocer que en algunos casos se pueden comportar como un cuerpo extraño y producir reacciones locales como consecuencia de la irritación crónica. Debemos de estar atentos ante esta posibilidad en caso de implantes en ambos canalículos del mismo ojo. Estas complicaciones nos obligan a realizar una cirugía para la extracción de dichos tapones y, como en esta paciente, además del granuloma formado.

\section{BIBLIOGRAFÍA}

1. Owens H, Phillips J. Spreading of the tears after a blink: velocity and stabilization time in healthy eyes. Cornea 2001; 20: 484-487.

2, Shalaby O, Sanz AI, Oroza MA, Rivas L, Murube J. Cambios en la superficie ocular en pacientes con queratoconjuntivitis seca antes y después del parche punctal. Arch Soc Esp Oftalmol 1998; 73: 521-526.

3. Musadiq M, Mukherji S, Sandramouli S. Pyogenic granuloma following silicone punctal plugs: report of two cases. Orbit 2005; 24: 149-151.

4. Yuen KS, Cheng AC, Chan WM. Pyogenic granulomas after silicone punctal plugs: a clinical and histopathologic study. Am J Ophthalmol 2005; 140: 963-964.

5. Chou TY, Perry HD, Donnenfeld ED. Pyogenic granuloma formation following placement of the Medennium SmartPLUG punctum plug. Cornea 2006; 25: 493-495. 